\title{
Electroacupuncture combined with mosapride alleviates symptoms in diabetic patients with gastroparesis
}

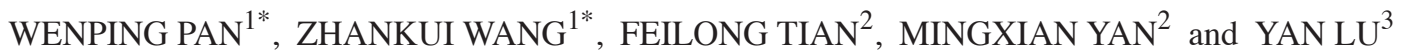 \\ Departments of ${ }^{1}$ Rheumatology and Immunology and ${ }^{2}$ Gastroenterology, Qianfoshan Hospital Affiliated \\ to Shandong University, Jinan, Shandong 250014; ${ }^{3}$ Department of Acupuncture and Moxibustion, \\ Shandong College of Traditional Chinese Medicine, Jinan, Shandong 250355, P.R. China
}

Received October 23, 2015; Accepted November 10, 2016

DOI: 10.3892/etm.2017.4139

\begin{abstract}
The present study compared the clinical effectiveness of electroacupuncture (EA), monotherapy and combination therapy involving the administration of EA and mosapride in diabetic patients with severe or mild symptoms suggestive of gastroparesis. A total of 56 patients with type 2 diabetes who had symptoms suggestive of gastroparesis for $>3$ months were divided into two groups according to the Gastroparesis Cardinal Symptom Index (GCSI) score, including 33 in the mild group (GCSI score <3.5) and 23 in the severe group (GCSI score $\geq 3.5$ ). Initially, all patients received EA monotherapy for 14 days. An effective response was defined as a reduction of the overall baseline GCSI score by $>25 \%$ after treatment. The non-responding patients then received a combination treatment with EA and mosapride. Gastric emptying was assessed by the ${ }^{13} \mathrm{C}$-octanoic acid breath test at the beginning and end of each treatment session. Two patients in the severe group dropped out of the study during the initial treatment session. The results revealed that 34 early-responding patients (30 from the mild group and 4 from the severe group) treated with EA monotherapy, and 20 non-early-responding patients receiving combination therapy with EA and mosapride showed clinically significant improvements. Analysis of data from the mild subgroup demonstrated that EA treatment specifically improved symptoms of nausea, vomiting, stomach fullness, excessive fullness and bloating. There was no statistically significant difference in the gastric half-emptying time among patients prior to and after EA monotherapy. These preliminary results suggested that EA may be an option for improving mild symptoms in
\end{abstract}

Correspondence to: Professor Mingxian Yan, Department of Gastroenterology, Qianfoshan Hospital Affiliated to Shandong University, 16766 Jingshi Road, Jinan, Shandong 250014, P.R. China E-mail:ming-xian.yan@163.com

\section{*Contributed equally}

Key words: electroacupuncture, mosapride, diabetes mellitus, gastroparesis patients with diabetic gastroparesis, whereas combination therapy involving EA and pharmaceutics is required in patients with severe symptoms.

\section{Introduction}

Diabetic gastroparesis is a common and debilitating disease affecting millions of patients with diabetes mellitus worldwide (1). Up to $50 \%$ of patients with type 1 or type 2 diabetes may suffer from gastroparesis, which can occur as a result of nutritional deficiency, poor glycemic control and psychological distress. Gastroparesis affects the quality of life of the patient and the management of diabetes (1-3). Current pharmacological therapies available for the treatment of gastroparesis have low effectiveness in drug resistant and non-responding patients.

In previous years, electroacupuncture (EA) has been widely used to treat patients with symptoms suggestive of gastroparesis (4-8). EA has potential benefits for the control of disorders of gastrointestinal motility in a more systematic way compared with other alternative therapies (9-12). Three small, randomized pilot studies demonstrated that EA effectively alleviated the symptoms of diabetic gastroparesis; however, the overall efficiency was not satisfactory $(4,6,7)$. The possible cause of the contrasting results may be a result of a failure to categorize the patients according to the severity of their symptoms (4,6-8). A study by Wang (5) showed that the effect of acupuncture was superior to that of drug treatment (with respect to domperidone) in terms of symptom alleviation, with a total efficacy rate of $94 \%$. However, the abovementioned study did not analyze the association between the severity of symptoms and the efficiency of the treatment. At present, detailed information regarding the treatment with EA has yet to be provided. The present study investigated whether EA is effective in patients with severe symptoms suggestive of gastroparesis and evaluated the effects of combination therapy of EA and mosapride.

\section{Materials and methods}

Patients. A total of 56 patients with type 2 diabetes mellitus who had symptoms suggestive of gastroparesis for $>3$ months, and had not received treatment with prokinetics or other 


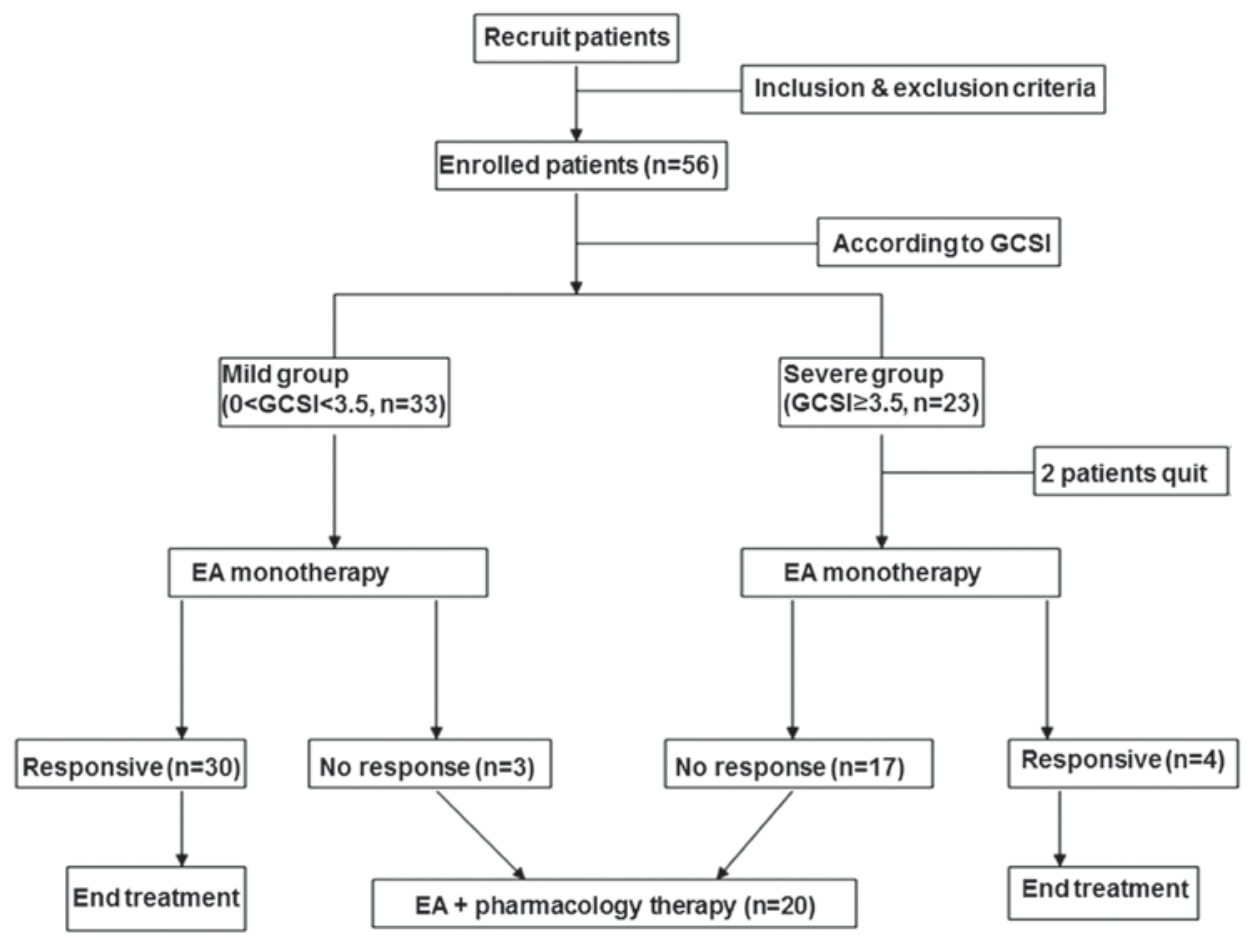

Figure 1. Study flowchart to show the movement of patients throughout the study. GCSI, Gastroparesis Cardinal Symptom Index; EA, electroacupuncture.

medications that may affect gastrointestinal motility in the previous 3 weeks, were enrolled in the present study. All the patients were recruited from clinical departments or the endocrinopathy and gastroenteropathy ward of Qianfoshan Hospital Affiliated to Shandong University (Jinan, China) from January 2011 to May 2012. Insulin was administered to patients if required. Endoscopy or abdominal X-ray examination was performed to exclude patients with primary gastrointestinal diseases if necessary. None of the patients had previously undergone surgical treatment on the gastrointestinal tract. The patients whose symptoms were severe enough for the clinician to consider nutritional support or other strategy, and those with severe complications (end-stage renal disease, coronary artery disease, stroke, cancer or lung disease) were also excluded from the study. Patient safety was evaluated by a daily assessment of adverse events. All patients received a daily physical examination from the commencement of the trial, and were continuously monitored by telephone interview for 7 days after treatment. The study protocol was approved by the Ethics Review Committee of Qianfoshan Hospital Affiliated to Shandong University. Written informed consent was obtained from all patients or their families.

Gastroparesis Cardinal Symptom Index (GCSI) assessment. GCSI, a validated questionnaire consisting of 3 subscales, was used to assess the predominant symptoms associated with gastroparesis, including: Postprandial fullness/early satiety, nausea/vomiting and bloating. GCSI total scores ranged between 0 and 5 , with a higher score indicating greater symptom severity. Symptom severity was classified as mild (GCSI score $<3.5$ ) or severe (GCSI score $\geq 3.5)(13,14)$. The patients were divided into two groups according to their GCSI scores as follows: i) Mild group ( $n=33)$ and ii) severe group $(n=23)$. However, 2 patients from the severe group dropped out of the study during the initial treatment session; therefore, only 21 patients from the severe group completed the study. In the first phase of the study, all patients received EA treatment once a day for 14 consecutive days. Individual and total GCSI scores were recorded each day in the morning prior to treatment. At the end of the 14-day treatment period, patients were considered to be non-responsive if the severity score was not reduced by $>25 \%$ from the baseline, and were then transferred to the second phase for combination therapy (EA and mosapride tablet) for a further 14 days (Fig. 1). The average frequency of vomiting of every patient on each day during each treatment session was also evaluated.

EA and mosapride administration. All acupuncture technicians had clinical and educational experience of $>10$ years, and had attained the corresponding certifications. Zusanli (ST36) and Neiguan (PC6) were selected as acupuncture points, as they have been reported to be effective stimulating points for improving gastroparesis (9-12). The acupuncture needle was inserted into the points, and stimulation was performed by acupuncture needles using an electroacupuncture apparatus (model G6805-2A; Shanghai Huayi Medical Instrument Factory, Shanghai, China) at a frequency of $25 \mathrm{~Hz}$ and an amplitude of 10-30 mA for a tolerable stimulation. Each daily treatment session lasted for $30 \mathrm{~min}$. Mosapride, a drug shown to effectively reduce the symptoms of diabetic gastroparesis, was selected for the combination therapy (15). Mosapride (5 mg per tablet; Sumitomo Pharmaceutical Suzhou Co., Suzhou, China) was orally administered at a dose of $5 \mathrm{mg} 3$ times a day for 2 consecutive weeks.

${ }^{13} \mathrm{C}$-octanoic acid breath test. Gastric emptying was assessed by the ${ }^{13} \mathrm{C}$-octanoic acid breath test $(16,17)$. All patients fasted overnight ( $\geq 8 \mathrm{~h}$ ) and consumed a standard test meal, consisting 
Table I. Patient demographics prior to treatment.

\begin{tabular}{lccr}
\hline Characteristics & Mild group $(\mathrm{n}=33)$ & Severe group $(\mathrm{n}=23)$ & P-value \\
\hline Age (years) & $53.6 \pm 11.2$ & $60.5 \pm 9.2$ & $>0.05$ \\
Gender (male/female) & $11 / 10$ & $11 / 8$ & $>0.05$ \\
Diabetes history (years) & $9.7 \pm 3.1$ & $11.9 \pm 7.7$ & $>0.05$ \\
Mean body mass index $\left(\mathrm{kg} / \mathrm{m}^{2}\right)$ & $27.9 \pm 5.6$ & $27.7 \pm 6.1$ & $>0.05$ \\
HbA1c $(\%)$ & $6.5 \pm 0.5$ & $6.7 \pm 1.0$ & $>0.05$ \\
Gastric half-emptying time $(\mathrm{min})$ & $126.3 \pm 28.2$ & $140.3 \pm 19.2$ & $>0.05$
\end{tabular}

Values are expressed as the mean \pm standard deviation. Depending on symptom severity, patients were stratified into the mild (GCSI score $<3.5$; $\mathrm{n}=33$ ) and the severe (GCSI score $\geq 3.5 ; \mathrm{n}=23$ ) groups. HbA1c, glycated hemoglobin; GCSI, Gastroparesis Cardinal Symptom Index .

of a raw egg mixed with $100 \mathrm{mg}{ }^{13} \mathrm{C}$-octanoic acid $\left(99 \%{ }^{13} \mathrm{C}\right)$ fried into an omelet and served with 2 pieces of white bread (420 kcal) and $150 \mathrm{ml}$ plain water. Each test meal was consumed within $10 \mathrm{~min}$. The ${ }^{13} \mathrm{C}$ concentration was measured by isotope ratio mass spectrometry (Breath MAT plus; Finnigan GmbH, Bremen, Germany) prior to the test meal and every $15 \mathrm{~min}$ for $4 \mathrm{~h}$ after the meal. Changes in ${ }^{13} \mathrm{C}$ concentrations at each time-point from baseline were analyzed to assess the gastric half-emptying time.

Statistical analysis. All evaluations were performed using SPSS statistical software (version 17.0; SPSS, Inc., Chicago, IL, USA). Non-parametric tests were performed by means of the Mann-Whitney U-test. Parametric tests used included the Student's $t$-test. All values are presented as the mean \pm standard deviation. $\mathrm{P} \leq 0.05$ was considered to indicate a statistically significant difference. All analyses pertaining to efficacy were based on two-sided tests.

\section{Results}

Patient demographics and gastric half-emptying. The patient demographics of all 56 patients included in the present study and their baseline characteristics were summarized in Table I. With the exception of the severity of symptoms at baseline (referring to GCSI total and individual scores), there were no differences between the two groups in terms of patient demographics (age, gender and body mass index) and clinical characteristics (diabetes duration and glycated hemoglobin). The gastric half-emptying time of patients was not significantly different between the mild and severe groups. No adverse events were observed during or after the trial.

EA or mosapride monotherapy alleviates symptoms and significantly reduces GCSI total scores in mild diabetic gastroparesis, while combination therapy is necessary for patients with severe symptoms. To assess the efficacy of EA monotherapy and combination therapy on diabetic gastroparesis, a GCSI assessment was performed. A total of 54 patients completed the study, including all 33 patients in the mild group and 21 patients in the severe group. Two patients in the severe group were dropped of the study due to severe vomiting that required medication during the first treatment session. These patients were aged 68 and 72 years, respectively, which was greatly above the average age of the treatment group. In addition, the diabetic duration of these patients was much longer than the average course. In the mild group, EA treatment resulted in significantly reduced GCSI total scores at the end of week $2(\mathrm{P}=0.016)$. Furthermore, the average reduction of the GCSI total score was $>25 \%$. In addition, analysis of the severe group showed that EA treatment failed to alleviate the symptoms of gastroparesis. There were 20 patients with no response to EA treatment ( 3 from the mild subgroup and 17 from the severe subgroup), and these patients achieved significantly improved GCSI total scores after combination therapy $(\mathrm{P}=0.013$; Table II). By analysis of categorical outcomes, numerical improvements were obtained for all individual scores; however, statistical significance was only observed in individual symptom scores for nausea, vomiting, stomach fullness, excessive fullness and bloating (Fig. 2). Although EA treatment did not alleviate all symptoms experienced by the severe group, it was beneficial to individual symptom scores of vomiting, stomach fullness and excessive fullness. After 6 days of treatment, in the mild group the GCSI total scores were observed to be reduced, and the highest efficacy was achieved on day 10. For non-responding patients, the scores began to improve on day 4 and plateaued on day 8 after the initiation of the combination therapy (Fig. 3). These results indicated that EA monotherapy alleviated symptoms and resulted in significantly reduced GCSI total scores in patients with mild diabetic gastroparesis, while combination therapy was necessary for patients with severe symptoms.

EA treatment and combination with mosapride reduces the frequency of vomiting in patients with mild and severe diabetic gastroparesis, respectively. To determine the effect of EA and mosapride on vomiting, the frequency of vomiting was assessed during the EA and combination treatment periods. The frequency of vomiting on each day experienced by mild and non-responding patients after treatment were significantly reduced compared with those prior to treatment $(\mathrm{P}=0.04$ and $\mathrm{P}=0.02$, respectively; Fig. 4). These results suggested that EA mono-treatment reduced the frequency of vomiting in patients with mild diabetic gastroparesis, while combined EA and mosapride treatment reduced the frequency of vomiting in patients not responding, which mainly comprised those with severe diabetic gastroparesis. 
Table II. Parameters prior to and after treatment.

\begin{tabular}{|c|c|c|c|c|c|c|c|c|c|}
\hline \multirow[b]{2}{*}{ Parameter } & \multicolumn{3}{|c|}{ Mild subgroup ( $\mathrm{n}=33$ ) } & \multicolumn{3}{|c|}{ Severe subgroup $(n=21)$} & \multicolumn{3}{|c|}{ Non-responding subgroup ${ }^{a}(n=20)$} \\
\hline & Baseline & At 2 weeks & P-value & Baseline & At 2 weeks & P-value & At 2 weeks & At 4 weeks & P-value \\
\hline \multicolumn{10}{|l|}{ GCSI score } \\
\hline Total & $2.25 \pm 0.54$ & $1.25 \pm 0.19$ & 0.016 & $3.96 \pm 0.60$ & $3.58 \pm 0.52$ & $>0.05$ & $4.16 \pm 0.36$ & $3.07 \pm 0.49$ & 0.013 \\
\hline Nausea & $2.53 \pm 0.69$ & $1.42 \pm 0.34$ & $<0.001$ & $4.01 \pm 0.39$ & $3.58 \pm 0.23$ & $>0.05$ & $4.21 \pm 0.56$ & $3.23 \pm 0.12$ & 0.027 \\
\hline Retching & $2.63 \pm 0.64$ & $2.21 \pm 0.32$ & $>0.05$ & $4.03 \pm 0.54$ & $3.71 \pm 0.23$ & $>0.05$ & $3.98 \pm 0.34$ & $3.52 \pm 0.14$ & $>0.05$ \\
\hline Vomiting & $2.01 \pm 0.62$ & $0.63 \pm 0.34$ & $<0.001$ & $3.71 \pm 0.62$ & $2.99 \pm 0.18$ & 0.040 & $3.64 \pm 0.12$ & $2.44 \pm 0.33$ & $<0.001$ \\
\hline Stomach fullness & $2.75 \pm 0.63$ & $1.89 \pm 0.41$ & 0.038 & $4.56 \pm 0.54$ & $4.05 \pm 0.58$ & 0.047 & $4.62 \pm 0.34$ & $3.70 \pm 0.64$ & 0.033 \\
\hline Early satiety & $2.75 \pm 0.54$ & $2.37 \pm 0.26$ & $>0.05$ & $3.78 \pm 0.79$ & $3.49 \pm 0.68$ & $>0.05$ & $4.08 \pm 0.32$ & $3.61 \pm 0.23$ & $>0.05$ \\
\hline Excessive fullness & $2.55 \pm 0.76$ & $1.33 \pm 0.66$ & $<0.001$ & $3.85 \pm 0.45$ & $3.21 \pm 0.32$ & 0.049 & $4.05 \pm 0.42$ & $3.04 \pm 0.54$ & 0.030 \\
\hline Loss of appetite & $2.08 \pm 0.86$ & $1.83 \pm 0.76$ & $>0.05$ & $3.92 \pm 0.44$ & $3.79 \pm 0.56$ & $>0.05$ & $4.10 \pm 0.33$ & $3.87 \pm 0.45$ & $>0.05$ \\
\hline Bloating & $2.05 \pm 0.63$ & $1.40 \pm 0.41$ & 0.036 & $4.12 \pm 0.72$ & $3.78 \pm 0.88$ & $>0.05$ & $3.99 \pm 0.15$ & $3.32 \pm 0.34$ & 0.035 \\
\hline Abdominal distension & $1.21 \pm 0.98$ & $0.98 \pm 0.72$ & $>0.05$ & $3.56 \pm 0.46$ & $3.41 \pm 0.65$ & $>0.05$ & $3.69 \pm 0.12$ & $3.24 \pm 0.15$ & $>0.05$ \\
\hline $\begin{array}{l}\text { Gastric half emptying } \\
\text { time (min) }\end{array}$ & $126.3 \pm 28.2$ & $113.8 \pm 27.3$ & 0.078 & $140.3 \pm 19.2$ & $133.8 \pm 13.2$ & 0.096 & $128.7 \pm 30.1$ & $98.8 .0 \pm 12.4$ & 0.018 \\
\hline
\end{tabular}

Values are expressed as the mean \pm standard deviation. Depending on symptom severity, patients were stratified into the mild (GCSI score $<3.5$; $\mathrm{n}=33$ ) and the severe (GCSI score $\geq 3.5 ; \mathrm{n}=21$ ) groups, who were treated by electroacupuncture. ${ }^{2}$ The non-responding group ( $\mathrm{n}=20$ ) comprised 3 patients from the mild and 17 patients from the severe group at day 14, who were treated for a further 14 days by combination therapy. GCSI, Gastroparesis Cardinal Symptom Index.

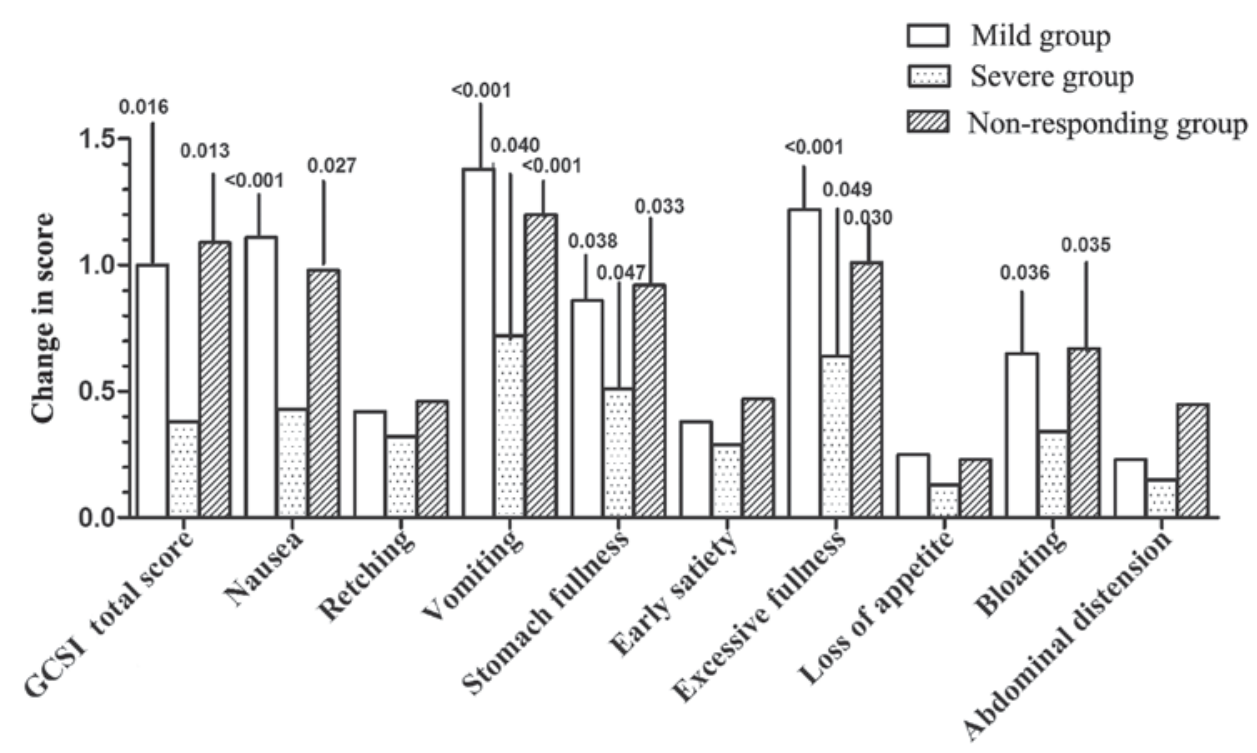

Figure 2. Changes of GCSI total scores and individual symptom scores prior to and after EA treatment for 14 days for the mild and severe groups or after an additional 14 days of EA and mosapride combination therapy for the non-responding group. GCSI, a validated questionnaire consisting of 3 subscales, was used to assess the major symptoms associated with gastroparesis: Postprandial fullness/early satiety, nausea/vomiting and bloating. GCSI total scores ranged between 0 and 5, with a higher score indicating greater symptom severity. Depending on symptom severity, patients were stratified into the mild (GCSI score $<3.5 ; n=33$ ) and the severe (GCSI score $\geq 3.5 ; n=21$ ) groups. The non-responding group ( $\mathrm{n}=20$ ) comprised 3 patients from the mild and 17 patients from the severe group at day 14, who were treated with combination therapy for a further 14 days. The numbers above the error bars are P-values, compared with those at baseline (for the mild and severe groups) or compared with those prior to the initiation of combination therapy (for the non-responding group). GCSI, Gastroparesis Cardinal Symptom Index; EA, electroacupuncture.

EA and mosapride combination treatment significantly reduces gastric half-emptying time. To evaluate gastric emptying time, the ${ }^{13} \mathrm{C}$-octanoic acid breath test was employed. The gastric half-emptying time in the mild group was slightly but not significantly decreased after EA (126.3 \pm 28.2 vs. $113.8 \pm 27.3 \mathrm{~min} ; \mathrm{P}=0.078)$. In the severe group, the gastric half-emptying time was not altered after 2 weeks of EA therapy $(140.3 \pm 19.2$ vs. $133.8 \pm 13.2 \mathrm{~min} ; \mathrm{P}=0.096)$; however, it was significantly decreased in the non-responding group following combination therapy $(128.7 \pm 30.1$ vs. $98.8 \pm 12.4 \mathrm{~min} ; \mathrm{P}=0.018)$. These results indicated that the gastric half-emptying time was significantly reduced by the combination treatment. 


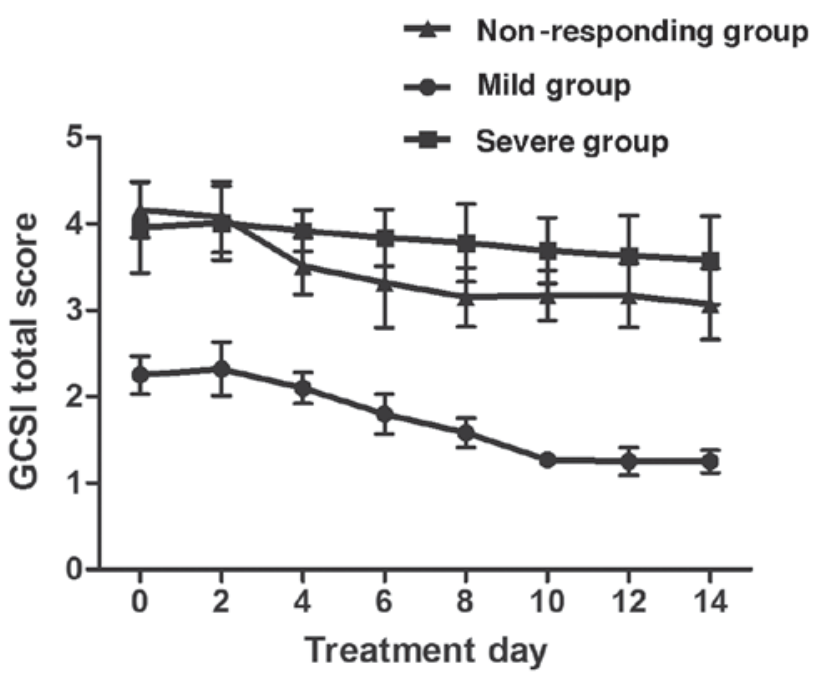

Figure 3. Time-dependent alleviation of Gastroparesis Cardinal Symptom Index total scores associated with gastroparesis by electroacupuncture or combination therapy. GCSI, a validated questionnaire consisting of 3 subscales, was used to assess the symptoms: Postprandial fullness/early satiety, nausea/vomiting and bloating. GCSI total scores ranged between 0 and 5 , with a higher score indicating a greater symptom severity. Depending on symptom severity, patients were stratified into the mild (GCSI score $<3.5$; $\mathrm{n}=33$ ) and the severe (GCSI score $\geq 3.5 ; \mathrm{n}=21$ ) groups. The non-responding group $(\mathrm{n}=20)$ comprised 3 patients from the mild and 17 patients from the severe group at day 14, who were treated with combination therapy for a further 14 days. GCSI, Gastroparesis Cardinal Symptom Index.

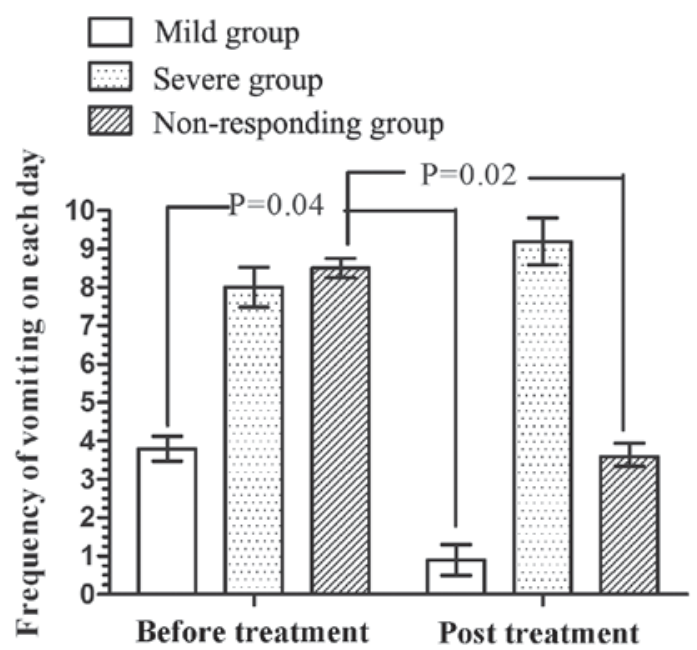

Figure 4. Average frequency of vomiting prior to and after treatment. The frequency of vomiting for each day on which patients experienced vomiting prior to and after electroacupuncture monotherapy for 14 days or combination therapy for an additional 14 days was recorded.

\section{Discussion}

Patients with diabetic gastroparesis may have mild or severe symptoms. EA or prokinetic agents are typically administered to alleviate the symptoms; however, patients may show different responses to the treatments. The findings of the present study suggested that EA significantly alleviates mild symptoms of gastroparesis and the results are consistent with those of previous studies $(4,5,7)$. Among the 34 patients with early responses to EA treatment, 30 had mild symptoms, while only 3 patients had severe symptoms. However, EA failed to relieve the symptoms in the severe group. The results indicated that a higher total GCSI score was associated with an unfavorable response to EA. However, non-responding patients, either from the mild or severe subgroup, showed significant alleviation of symptoms at the final evaluation after combination treatment. The 3 non-responding patients in the mild symptom group had a longer duration of upper gastrointestinal symptoms and a higher age compared with the remaining patients. However, whether the differences in diabetes history and age had a clinically significant effect cannot be concluded from the present study. Therefore, the treatment modality, namely combined treatment rather than EA therapy alone, should be selected based on the severity of the symptoms. Combination treatment involving EA and mosapride is an optimal treatment option for patients with diabetic gastroparesis with severe symptoms.

EA has been reported to improve or normalize gastric dysrhythmia via the vagal pathway, and to increase plasma pancreatic polypeptide levels in humans $(4,7)$. Acupuncture restores the balance of parasympathetic and sympathetic nerves, the disturbance of which is considered to be the sole cause of gastrointestinal dysmotility in diabetes mellitus $(18,19)$. In addition, EA has been demonstrated to modulate gastric motility through regulating the expression of transient receptor potential vanilloid $1, \mathrm{a} \mathrm{Ca}^{2+}$-permeable non-selective cation channel that has important physiological functions in peripheral and central nervous systems $(20,21)$. In recent decades, numerous studies on patients and animals have been performed to elucidate the effects of acupuncture on gastric myoelectrical activity as well as the underlying mechanisms (4-12,18-24). However, the exact mechanisms by which acupuncture improves gastric motility and dyspeptic symptoms have yet to be elucidated.

Although acupuncture has been reported to accelerate gastric emptying in animals and patients $(6,7,10,25,26)$, the data of the present study are not fully consistent with this. Gastroparesis symptoms may be incited by various etiologies, including functional dyspepsia and chronic gastritis. Delayed gastric half-emptying has been observed in $\sim 35 \%$ of patients with functional dyspepsia $(27,28)$. In the present study, a cohort of patients was selected based on clinical symptoms suggestive of gastroparesis. Therefore, the patients enrolled in the present study cannot be assumed to have delayed gastric half-emptying times. Previous studies have demonstrated an insignificant correlation between the gastric emptying rate and upper gastrointestinal symptom severity or symptom alleviation in patients with diabetes $(29,30)$, and the gastric emptying rate is reportedly also affected by plasma glucose levels and gender (31). In the present study, no statistically significant difference was detected in terms of gastric emptying rate prior to and following EA mono-treatment in all patients, which may be due to the small sample size of the study and the aforementioned factors.

Patients with gastroparesis display symptoms including nausea, vomiting, excessive fullness and bloating. In order to investigate the different effects of EA on specific symptoms, 
GCSI individual scores were evaluated prior to and after EA treatment in the present study. In the present study, significant alleviation was observed in nausea, vomiting, stomach fullness, excessive fullness and bloating after EA treatment in early-responding patients, while other symptoms were only slightly alleviated. Although patients in the severe group failed to respond to EA according to GCSI individual scores, they experienced significant alleviation in levels of vomiting, stomach fullness and excessive fullness. Previous reviews also showed evidence of the efficacy of acupuncture in reducing nausea and vomiting $(32,33)$.

In addition, the present study found that EA did not have any effect after 1 day of treatment, and significant alleviation of symptoms appeared to occur between days 7 and 9. This indicated that repeated treatment with EA may be required to achieve a satisfactory effect in clinical practice. By contrast, an extended treatment time may not be beneficial. Between days 7 and 9, the effects reached a peak and fluctuated slightly between days 9 and 14. Therefore, excessively long EA treatment is not necessary. Of note, in EA and mosapride combination therapy, the onset and peak time were advanced. This indirectly suggests that EA may enhance the effects of mosapride. Therefore, it can be speculated that EA may enable a reduction in the dose of prokinetics in clinical practice. A previous study demonstrated the association between the use of another prokinetic, domperidone, and sudden cardiac death at doses of $>30 \mathrm{mg} /$ day (34).

In conclusion, the present study suggested that EA combined with mosapride may be an optimal treatment for patients with severe diabetic gastroparesis symptoms, although EA alone is effective for alleviating mild symptoms. However, the sample size in the present study was not of sufficient size and further studies are required to investigate its optional use in clinical practice.

\section{Acknowledgements}

The authors thank their colleagues in various departments of Qianfoshan Hospital Affiliated to Shandong University and Shandong College of Traditional Chinese Medicine (Jinan, China) for their cooperation and assistance.

\section{References}

1. Maleki D, Locke GR III, Camilleri M, Zinsmeister AR, Yawn BP, Leibson C and Melton LJ III: Gastrointestinal tract symptoms among persons with diabetes mellitus in the community. Arch Intern Med 160: 2808-2816, 2000.

2. Bytzer P, Talley NJ, Leemon M, Young LJ, Jones MP and Horowitz M: Prevalence of gastrointestinal symptoms associated with diabetes mellitus: A population-based survey of 15,000 adults. Arch Intern Med 161: 1989-1996, 2001.

3. Camilleri M, Parkman HP, Shafi MA, Abell TL and Gerson L, American College of Gastroenterology; Clinical guideline: Management of gastroparesis. Am J Gastroenterol 108: 18-37, quiz 38, 2013.

4. Chang CS, Ko CW, Wu CY and Chen GH: Effect of electrical stimulation on acupuncture points in diabetic patients with gastric dysrhythmia: A pilot study. Digestion 64: 184-190, 2001.

5. Wang L: Clinical observation on acupuncture treatment in 35 cases of diabetic gastroparesis. J Tradit Chin Med 24: 163-165, 2004.

6. Xu S, Hou X, Zha H, Gao Z, Zhang Y and Chen JD: Electroacupuncture accelerates solid gastric emptying and improves dyspeptic symptoms in patients with functional dyspepsia. Dig Dis Sci 51: 2154-2159, 2006.
7. Wang CP, Kao CH, Chen WK, Lo WY and Hsieh CL: A single-blinded, randomized pilot study evaluating effects of electroacupuncture in diabetic patients with symptoms suggestive of gastroparesis. J Altern Complement Med 14: 833-839, 2008.

8. Liu S, Peng S, Hou X, Ke M and Chen JD: Transcutaneous electroacupuncture improves dyspeptic symptoms and increases high frequency heart rate variability in patients with functional dyspepsia. Neurogastroenterol Motil 20: 1204-1211, 2008.

9. Lin X, Liang J, Ren J, Mu F, Zhang M and Chen JD: Electrical stimulation of acupuncture points enhances gastric myoelectrical activity in humans. Am J Gastroenterol 92: 1527-1530, 1997.

10. Ouyang H, Yin J, Wang Z, Pasricha PJ and Chen JD: Electroacupuncture accelerates gastric emptying in association with changes in vagal activity. Am J Physiol Gastrointest Liver Physiol 282: G390-G396, 2002.

11. Gao X, Qiao Y, Jia B, Jing X, Cheng B, Wen L, Tan Q, Zhou Y, Zhu B and Qiao H: NMDA Receotor-Dependent synaptic activity in dorsal motor nucleus of vagus mediates the enhancement of gastric motility by stimulating ST36. Evid Based Complement Alternat Med 2012: 438460, 2012.

12. Lee CH, Kim DK, Yook TH, Sasaki M and Kitamura N: Effectiveness of electroacupuncture at Zusanli(ST36) on the immunohistochemical density of enteroendocrine cells related to gastrointestinal function. J Acupunct Meridian Stud 5: 63-71, 2012.

13. Revicki DA, Rentz AM, Dubois D, Kahrilas P, Stanghellini V, Talley NJ and Tack J: Development and validation of a patient-assessed gastroparesis symptom severity measure: The Gastroparesis Cardinal Symptom Index. Aliment Pharmacol Ther 18: 141-150, 2003.

14. Revicki DA, Rentz AM, Dubois D, Kahrilas P, Stanghellini V, Talley NJ and Tack J: Gastroparesis Cardinal Symptom Index (GCSI): Development and validation of a patient reported assessment of severity of gastroparesis symptoms. Qual Life Res 13: 833-844, 2004

15. Talley NJ: Diabetic gastropathy and prokinetics. Am J Gastroenterol 98: 264-271, 2003.

16. Mansi C, Mekga P and Savarino V: Gastric emptying evaluation by 13C-octanoic acid breath test. Diabetes Nutr Metab 17: 43-46, 2004.

17. Usai Satta P, Scarpa M, Oppia F and Loriga F: 13C-octanoic acid breath test in function and organic disease: Critical review of literature. Eur Rev Med Pharmacol Sci 9 (5 Suppl 1): S9-S13, 2005.

18. Takahashi T: Mechanism of acupuncture on neuromodulation in the gut: A review. Neuromodulation 14: 8-12, 2011.

19. Takahashi T: Effect and mechanism of acupuncture on gastrointestinal diseases. Int Rev Neurobiol 111: 273-294, 2013.

20. Abraham TS, Chen ML and Ma SX: TRPV1 expression in acupuncture points: Response to electroacupuncture stimulation. J Chem Neuroanat 41: 129-136, 2011.

21. Wang SJ, Yang HY and Xu GS: Acupuncture alleviates colorectal hypersensitivity and correlates with the regulatory mechanism of TrpV1 and p-ERK. Evid Based Complement Alternat Med 2012: 483123, 2012.

22. Schneider A, Steitberger K and Joos S: Acupunture treatment in gastrointestinal disease: A systematic review. World J Gastroenterol 13: 3417-3424, 2007.

23. Yin J and Chen JD: Gastrointestinal motility disorders and acupuncture. Auton Neurosci 157: 31-37, 2010.

24. Fukuta H, Koshita M, Nakamura E, Nakamura H, Yamada A, Kawase Y, Ishigami T, Kurono Y, Iino S and Suzuki H: Acupuncture modulates mechanical responses of smooth muscle produced by transmural nerve stimulation in gastric antrum of genetically hyperglycemic rats. J Smooth Muscle Res 45: $167-185,2009$.

25. Iwa M, Nakade Y, Pappas TN and Takahashi T: Electroacupuncture elicits dual effects: Stimulation of delayed gastric emptying and inhibition of accelerated colonic transit induced by restraint stress in rats. Dig Dis Sci 51: 1493-1500, 2006.

26. Tabosa A, Yamamura Y, Forno ER and Mello LE: A comparative study of the effects of electroacupuncture and moxibustion in the gastrointestinal motility of the rat. Dig Dis Sci 49: 602-610, 2004.

27. Lorena SL, Tinois E, Brunetto SQ, Camargo EE and Mesquita MA: Gastric emptying and intragastric distribution of a solid meal in functional dyspepsia: Influence of gender and anxiety. J Clin Gastroenterol 38: 230-236, 2004. 
28. Stanghellini V, De Giorgio R, Barbara G, Cogliandro R, Tosetti C, De Ponti F and Corinaldesi R: Delayed gastric emptying in functional dyspepsia. Curr Treat Options Gastroenterol 7: 259-264, 2004.

29. Samsom M, Vermeijden JR, Smout AJ, Van Doorn E, Roelofs J, Van Dam PS, Martens EP, Eelkman-Rooda SJ and Van Berge-Henegouwen GP: Prevalence of delayed gastric emptying in diabetic patients and relationship to dyspeptic symptoms: A prospective study in unselected diabetic patients. Diabet Care 26: 3116-3122, 2003.

30. Pasricha PJ, Colvin R, Yates K, Hasler WL, Abell TL, Unalp-Arida A, Nguyen L, Farrugia G, Koch KL, Parkman HP, et al: Characteristics of patients with chronic unexplained nausea and vomiting and normal gastric emptying. Clin Gastroenterol Hepatol 9: 567-576.e1-4, 2011.

31. Parkman HP, Yates K, Hasler WL, Nguyen L, Pasricha PJ, Snape WJ, Farrugia G, Koch KL, Abell TL, McCallum RW, et al: Clinical features of idiopathic gastroparesis vary with sex, body mass, symptom onset, delay in gastric emptying, and gastroparesis severity. Gastroenterology 140: 101-115, 2011.
32. Schneider A, Löwe B and Streitberger K: Perception of bodily sensation as a predictor of treatment response to acupuncture for postoperative nausea and vomiting prophylaxis. J Altern Complement Med 11: 119-125, 2005.

33. Lee A and Done ML: The use of nonpharmacologic techniques to prevent postoperative nausea and vomiting: A meta-analysis. Anesth Analg 88: 1362-1369, 1999.

34. Janssen P, Harris MS, Jones M, Masaoka T, Farré R, Törnblom H, Van Oudenhove L, Simrén M and Tack J: The relation between symptom improvement and gastric emptying in the treatment of diabetic and idiopathic gastroparesis. Am J Gastroenterol 108: 1382-1391, 2013. 